\title{
A Simulation Evaluation of the Maximum Approximate Composite Marginal Likelihood (MACML) Estimator for Mixed Multinomial Probit Models
}

\author{
Chandra R. Bhat* \\ The University of Texas at Austin \\ Department of Civil, Architectural and Environmental Engineering \\ 1 University Station, C1761 \\ Austin, TX 78712-0278 \\ Phone: (512) 471-4535, Fax: (512) 475-8744 \\ Email: bhat@mail.utexas.edu \\ Raghuprasad Sidharthan \\ The University of Texas at Austin \\ Department of Civil, Architectural and Environmental Engineering \\ 1 University Station, C1761 \\ Austin, TX 78712-0278 \\ Phone: (512) 471-4535, Fax: (512) 475-8744 \\ Email: raghu@mail.utexas.edu \\ *corresponding author
}

Original: July 13, 2010

Revised: January 18, 2011

$2^{\text {nd }}$ Revision: April 18, 2011 


\section{ABSTRACT}

This paper evaluates the ability of the maximum approximate composite marginal likelihood (MACML) estimation approach to recover parameters from finite samples in mixed crosssectional and panel multinomial probit models. Comparisons with the maximum simulated likelihood (MSL) estimation approach are also undertaken. The results indicate that the MACML approach recovers parameters much more accurately than the MSL approach in all model structures and covariance specifications. The MACML inference approach also estimates the parameters efficiently, with the asymptotic standard errors being, in general, only a small proportion of the true values. As importantly, the MACML inference approach takes only a very small fraction of the time needed for MSL estimation. In particular, the results suggest that, for the case of five random coefficients, the MACML approach is about 50 times faster than the MSL for the cross-sectional random coefficients case, about 15 times faster than the MSL for the panel inter-individual random coefficients case, and about 350 times or more faster than the MSL for the panel intra- and inter-individual random coefficients case. As the number of alternatives in the unordered-response model increases, one can expect even higher computational efficiency factors for the MACML over the MSL approach. Further, as should be evident in the panel intra- and inter-individual random coefficients case, the MSL is all but practically infeasible when the mixing structure leads to an explosion in the dimensionality of integration in the likelihood function, but these situations are handled with ease in the MACML approach. It is hoped that the MACML procedure will spawn empirical research into rich model specifications within the context of unordered multinomial choice modeling, including autoregressive random coefficients, dynamics in coefficients, space-time effects, and spatial/social interactions.

Keywords: mixed multinomial probit, composite marginal likelihood, maximum simulated likelihood, discrete choice models, unordered-response models, panel data. 


\section{INTRODUCTION}

Consider the following random-coefficients formulation in which the utility that an individual $q$ associates with alternative $i$ is given by:

$$
U_{q i}=\boldsymbol{\beta}_{q}^{\prime} \boldsymbol{x}_{q i}+\varepsilon_{q i}
$$

where $\boldsymbol{x}_{q i}$ is a $(K \times 1)$-column vector of exogenous attributes, and $\boldsymbol{\beta}_{q}$ is an individual-specific $(K \times 1)$-column vector of corresponding coefficients that is a realization from a multivariate normal density function with mean vector $\boldsymbol{b}$ and covariance matrix $\boldsymbol{\Omega}$. $\varepsilon_{q i}$ is assumed to be an independently and identically distributed (across alternatives and across individuals) error term, which is also independent of the covariate vector $\boldsymbol{x}_{q i}$. If $\varepsilon_{q i}$ is normally distributed with a mean zero and variance of one-half, then the likelihood contribution of individual $q$ who chooses alternative $m$ is:

$$
L_{q}=\int_{\boldsymbol{\beta}=-\infty}^{\infty}\left\{\int_{\lambda=-\infty}^{\infty}\left(\prod_{i \neq m}\left[\Phi\left\{\left[-\sqrt{2}\left(\boldsymbol{\beta}^{\prime} \boldsymbol{z}_{\text {qim }}\right)\right]+\lambda\right\}\right]\right) \phi(\lambda) d \lambda\right\} f(\boldsymbol{\beta} \mid \boldsymbol{b}, \boldsymbol{\Omega}) \boldsymbol{d} \boldsymbol{\beta},
$$

where $\boldsymbol{z}_{q i m}=\boldsymbol{x}_{q i}-\boldsymbol{x}_{q m}, \Phi($.$) is the univariate cumulative distribution function and \phi($.$) is the$ univariate normal density function. In the case of panel data, the utility structure may be written with the inclusion of choice occasion $t$ as:

$$
U_{q i t}=\boldsymbol{\beta}_{q}^{\prime} \boldsymbol{x}_{q i t}+\varepsilon_{q i t} \cdot
$$

In this case, the individual likelihood contribution of individual $q$ choosing alternative $m_{t}$ at choice occasion $t$ when $\varepsilon_{\text {qit }}$ is normally distributed, is:

$$
L_{q}=\int_{\boldsymbol{\beta}=-\infty}^{\infty} \prod_{t=1}^{T}\left[\int_{\lambda=-\infty}^{\infty}\left(\prod_{i \neq m_{t}}\left[\Phi\left\{\left[-\sqrt{2}\left(\boldsymbol{\beta}^{\prime} \boldsymbol{z}_{q i \boldsymbol{m}_{t} t}\right)\right]+\lambda\right\}\right]\right) \phi(\lambda) d \lambda\right] f(\boldsymbol{\beta} \mid \boldsymbol{b}, \boldsymbol{\Omega}) \boldsymbol{d} \boldsymbol{\beta},
$$

where $\boldsymbol{z}_{q i m_{t} t}=\boldsymbol{x}_{q i t}-\boldsymbol{x}_{q m t}$.

Finally, in the case of panel data, and when the random coefficients have both an intra-individual and inter-individual random component (see Bhat and Castelar, 2002; Bhat and Sardesai, 2006, Hess and Rose, 2009), the utility structure may be written as: 
$U_{q i t}=\boldsymbol{\beta}_{q t}^{\prime} \boldsymbol{x}_{q i t}+\varepsilon_{q i t}$,

where $\boldsymbol{\beta}_{q t}=\boldsymbol{\beta}_{q}+\tilde{\boldsymbol{\beta}}_{q t}, \boldsymbol{\beta}_{q} \sim N(\boldsymbol{b}, \boldsymbol{\Omega}), \tilde{\boldsymbol{\beta}}_{q t} \sim N(0, \tilde{\boldsymbol{\Omega}})$.

In this case, when $\varepsilon_{q i t}$ is normally distributed,

$$
L_{q}=\int_{\boldsymbol{\beta}=-\infty}^{\infty}\left[\prod_{t=1}^{T}\left(\int_{\tilde{\boldsymbol{\beta}}=-\infty}^{\infty}\left\{\int_{\lambda=-\infty}^{\infty}\left(\prod_{i \neq m_{t}}\left[\Phi\left\{\left[-\sqrt{2}\left(\boldsymbol{\beta}^{\prime}+\tilde{\boldsymbol{\beta}}^{\prime}\right) z_{q i m_{t}}\right]+\lambda\right\}\right]\right) \phi(\lambda) d \lambda\right\} f(\tilde{\boldsymbol{\beta}} \mid \tilde{\boldsymbol{\Omega}}) d \tilde{\boldsymbol{\beta}}\right)\right] f(\boldsymbol{\beta} \mid \boldsymbol{b}, \boldsymbol{\Omega}) \boldsymbol{d} \boldsymbol{\beta}
$$

The likelihood contribution of individual $q$ in Equations (2), (4), and (6) entails the evaluation of an analytically intractable function with multidimensional integrals. This has led to the development of various simulation techniques in high dimensions as part of a maximum simulated likelihood (MSL) estimation approach. Unfortunately, for many practical situations, the computational cost to ensure good asymptotic MSL estimator properties can be prohibitive and literally infeasible (in the context of the computation resources available and the time available for estimation) as the number of dimensions of integration increases.

In a companion paper, Bhat (2011a) proposed the use of an alternative maximum approximate composite marginal likelihood (MACML) estimator within the class of frequentist estimators for the estimation of multinomial probit (MNP) models. Bhat's MACML estimator is based solely on univariate and bivariate cumulative normal distribution evaluations, regardless of the dimensionality of integration. This should substantially reduce computation time compared to more cumbersome simulation techniques to evaluate multidimensional integrals. At the same time, the MACML estimator retains the properties of being consistent and asymptotically normally distributed.

The specific objectives of this study are motivated by the discussion above. The first objective is to examine the ability of the MACML estimator to recover parameters from finite samples in mixed cross-sectional and panel multinomial probit models. We use simulated data sets with known underlying model parameters to evaluate the MACML approach. The second, related, objective is to compare the performance of the MACML approach with the MSL approach in mixed MNP simulations when the MSL approach is feasible. In doing so, we examine the relative ability of the two approaches to recover parameters and the computation time of the two approaches. 
The rest of the paper is structured as follows. Section 2 presents the experimental design for the simulation experiments and Section 3 presents the results. Section 4 concludes the paper by highlighting important findings.

\section{EXPERIMENTAL DESIGN}

In the simulation set-up to examine the performance of the MSL and MACML inference approaches, we consider the case of five alternatives with five independent variables. For all the datasets generated in the experimental design, the values of each of the five independent variables for the alternatives are drawn from a standard univariate normal distribution. For the cross-sectional data set, we generate a sample of 5000 realizations of the five independent variables corresponding to 5000 individuals, while, for the panel data set, we generate a sample of 2500 realizations of the five independent variables corresponding to a situation where 500 individuals each have five choice occasions for a total of 2500 choice occasions. We allow random coefficients on all the five independent variables. This leads to a five-dimensional integral in the mixed model. In the subsequent three sections, we discuss the set-up for each of the following three cases in more detail: (1) cross-sectional random coefficients, (2) panel interindividual coefficients and (3) panel intra-individual and inter-individual random coefficients.

\subsection{Cross-Sectional Random Coefficients Model Structure}

In the cross-sectional case, the coefficient vector $\boldsymbol{\beta}_{q}$ for individual $q$ is assumed to be a realization from a multivariate normal distribution with a mean vector $\boldsymbol{b}=(1.5,-1,2,1,-2)$ and covariance matrix $\boldsymbol{\Omega}$. Two specifications for $\boldsymbol{\Omega}$ are considered. The first specification, which we label as the diagonal covariance specification, assumes independence among the random coefficients; that is, the matrix $\boldsymbol{\Omega}$ is assumed to be diagonal. This specification has been frequently used in the literature. The entries along the diagonal are set to the value of 1 in our experimental design. This first specification entails the estimation of five parameters in the covariance matrix. The second specification, which we label as the non-diagonal covariance specification, allows the random coefficients to be correlated. In this specification, we specify the matrix $\boldsymbol{\Omega}$ to be as follows: 


$$
\boldsymbol{\Omega}=\left[\begin{array}{ccccc}
1 & -0.50 & 0.25 & 0.75 & 0 \\
-0.50 & 1 & 0.25 & -0.50 & 0 \\
0.25 & 0.25 & 1 & 0.33 & 0 \\
0.75 & -0.50 & 0.33 & 1 & 0 \\
0 & 0 & 0 & 0 & 1
\end{array}\right]
$$

This positive definite non-diagonal specification involves the estimation of 10 covariance matrix parameters. Finally, values for the error terms $\varepsilon_{q i}(q=1,2, \ldots, Q ; i=1,2, \ldots, I)$ in Equation (1) are generated from a univariate normal distribution with a variance of 0.5 , leading to the mixed MNP model structure. The alternative with the highest utility for each observation is then identified as the chosen alternative. The above data generation process is undertaken 20 times with different realizations of the $\boldsymbol{\beta}_{q}$ vector and the error term $\varepsilon_{q i}$ to generate 20 different data sets each for the diagonal specification and the non-diagonal specification of the $\boldsymbol{\Omega}$ matrix.

The MSL and MACML estimators are applied to each data set to estimate data specific values of $\boldsymbol{b}$ and $\boldsymbol{L}$ ( $\boldsymbol{\Omega}=\boldsymbol{L} \boldsymbol{L}^{\prime}$, where $\boldsymbol{L}$ is the lower Cholesky decomposition of $\boldsymbol{\Omega}$; note that it is the Cholesky parameters that are estimated to ensure the positive definiteness of the variancecovariance matrix $\boldsymbol{\Omega}$ ). In the case of the diagonal covariance specification, $\boldsymbol{L}$ is also a diagonal matrix with entries of ' 1 ' along the diagonal. The MSL estimator is applied to each dataset 10 times with different (independent) draws for the random coefficients for each individual. This allows us to estimate the simulation error in the MSL case by computing the standard deviation of estimated parameters among the 10 different estimates on the same data set. Similarly, for the MACML approach, the approximation error is obtained by computing the standard deviation of estimated parameters among the 10 different estimates on the same data set by using different permutations to decompose the multivariate normal cumulative distribution (MVNCD) function into a product sequence of marginal and conditional probabilities (see Section 2.1 of Bhat, 2011a).

For the MSL estimation, we use draws from the Halton sequence for the random coefficients vector $\boldsymbol{\beta}_{q}$, because it is the most commonly used QMC sequence in the literature. While some other QMC systems have been shown to provide better results for a given number of draws, the Halton has the advantage of very easy generation. Thus, as indicated by Sandor and Train (2004), one can generate many more draws per individual of the Halton sequence than 
other QMC sequences for the same amount of time. Within the context of Halton draws, we experimented with different kinds of scramblings and randomizations of the Halton sequence (see Bhat, 2003 and Sivakumar et al., 2005 for a review of these scrambling and randomization techniques). The experiments indicated that the best performance was obtained using a procedure that combined Bratten-Weller scrambling with the Tuffin randomization, further enhanced by the random assignment of Halton dimensions to coefficients. Also, while a higher number of draws per individual (based on the combination scrambling/randomization discussed above) generally provided improved results, we used 250 draws per individual, which is more than what is typically used in most applications of the MSL procedure. Further, with a total of 400 total estimations for the cross-sectional random coefficients case (20 simulation runs for each of 10 different data samples for each of the diagonal and non-diagonal covariance case), an important factor was to keep the computation cost per estimation to a reasonable amount of time (even with 250 draws per individual, the total computer time for the 400 estimations was over 800 hours, as we discuss in more detail later). Finally, note that one has to integrate out the inner onedimensional integral over the scalar $\lambda$ that is distributed standard normal (see Equation (2)). While this integration can also be performed using QMC draws, we undertake this inner onedimensional integration using the more efficient hermite quadrature technique with 10 quadrature points.

For the MACML method, a single random permutation is generated for each individual (the random permutation varies across individuals, but is the same across iterations for a given individual), and the multivariate normal cumulative distribution (MVNCD) function is approximated using the resulting conditional probability sequence. We used different numbers of random permutations per individual to approximate the MVNCD function corresponding to the individual likelihood contribution. However, there was hardly any difference between using a single permutation and higher numbers of permutations, and hence we used a single permutation per individual (in one of the 400 estimations undertaken in the cross-sectional case, using two permutations per individual instead of a single permutation provided stability to the iterations).

\subsection{Panel Inter-Individual Random Coefficients}

As in the cross-sectional case, for the panel case too, we consider both a diagonal specification as well as a non-diagonal specification for the $\boldsymbol{\beta}_{q}$ random coefficient vector, with the mean vector 
and the covariance matrix of $\boldsymbol{\beta}_{q}$ identical to the cross-sectional case. The difference is that we generate only 500 vectors of coefficients, one vector for each of the 500 individuals. The same individual-specific coefficient vector is applied to all 5 choice occasions of the individual. The values for the error terms $\varepsilon_{\text {qit }}$ are generated from a univariate normal distribution with a variance of 0.5 , and the alternative with the highest utility is designated as the chosen alternative at each choice occasion for each individual.

The data generation process is undertaken 10 times with different sets of 500 realizations

of the $\boldsymbol{\beta}_{q}$ vector and 2500 realizations of the error term $\varepsilon_{q i t}$ to obtain 10 different data sets (we used fewer data samples and fewer total observations for the panel case compared to the crosssectional case because of the increased computational costs for panel data relative to crosssectional data). The MSL and MACML estimation procedures are applied to each data set. For the MSL approach, we decided to ignore simulation error and estimated only a single set of parameters for each data set using 250 Halton draws because of the computation time involved. Also, we observed during the MSL runs that the analytic gradient function was not returning accurate values consistent with the likelihood function for 10 quadrature points when integrating out $\lambda$ in Equation (4). This is not surprising, since the product across choice occasions of the same individual is now within the integration for $\lambda$. The net result was that the convergence process would get stuck because of the inaccuracy. So, we had to increase the accuracy of the gradient procedure by increasing the number of hermite quadrature points to 40 in the panel case. For the MACML case, we estimated the approximation error by estimating the model 10 times for each data set with different sets of permutations (as in the cross-sectional case). We tested the performance of the MACML method by using both a single permutation per individual as well as two permutations per individual, and found (as in the cross-sectional case) that the performance improvement was rather marginal.

\subsection{Panel Intra-Individual and Inter-Individual Random Coefficients}

This estimation involves the generation of the 500 vector realizations of coefficients for $\boldsymbol{\beta}_{q}$ as earlier from the multivariate normal distribution with a mean vector $\boldsymbol{b}$ and covariance $\boldsymbol{\Omega}$. In addition, 2,500 vectors of coefficients for $\tilde{\boldsymbol{\beta}}_{q t}$ (see Equation (5)) are generated from the multivariate normal distribution with a mean vector of 0 and covariance $\tilde{\boldsymbol{\Omega}}$. As in the earlier 
cases, we considered both a diagonal specification for $\boldsymbol{\Omega}$ and $\tilde{\boldsymbol{\Omega}}$, as well as non-diagonal specifications for both covariance matrices. The diagonal specification involved draws for $\boldsymbol{\beta}_{q}$

and $\tilde{\boldsymbol{\beta}}_{q t}$ from standard and independently normally distributions, while the non-diagonal covariance specification for $\boldsymbol{\beta}_{q}$ was the same as in Section 2.2 and the non-diagonal covariance specification for $\tilde{\boldsymbol{\beta}}_{q t}$ was as below:

$$
\widetilde{\boldsymbol{\Omega}}=\left[\begin{array}{ccccc}
1 & 0 & 0 & 0 & 0 \\
0 & 1 & 0.5 & 0.5 & 0.5 \\
0 & 0.5 & 1 & 0.5 & 0.5 \\
0 & 0.5 & 0.5 & 1 & 0.5 \\
0 & 0.5 & 0.5 & 0.5 & 1
\end{array}\right]
$$

The sum of $\boldsymbol{\beta}_{\boldsymbol{q}}$ and $\boldsymbol{\beta}_{\boldsymbol{q}}$ realizations are then applied to the independent variable vector for each individual's choice occasion to identify the alternative with highest utility. Everything else remains identical to Section 2.2.

\section{PERFORMANCE COMPARISON BETWEEN THE MSL AND MACML APPROACHES}

In this section, we first identify a number of performance measures and discuss how these are computed for the MSL approach and the MACML approach. The subsequent sections present the simulation and computational results.

\subsection{Performance Measures}

The steps discussed below for computing performance measures are for a specific correlation matrix pattern. We discuss the approach first for the cross-sectional random coefficients case, and then indicate the minor modifications for the two panel random coefficients cases.

\section{MSL Approach}

(1) Estimate the MSL parameter estimates for each data set $s(s=1,2, \ldots, 20)$ and for each of the 10 independent scrambled and randomized Halton draws, and obtain the time to obtain the convergent values and the standard errors. Obtain the mean time for convergence 
(TMSL) and standard deviation of convergence time across the 200 runs for each correlation pattern The 200 runs correspond to 10 runs for each of 20 data sets. The time to convergence includes the time to compute the covariance matrix of parameters and the corresponding parameter standard errors. All estimations are started with the true parameter values as the starting values. While multiple computers had to be used for the many different runs undertaken in this paper, all the run times were carefully scaled to the equivalent time on a desktop computer with 3GHz Quad core processor and 8GB of RAM. The scaling was based on extensive experimentation on different computers.

(2) For each data set $s$ and draw combination, estimate the standard errors (s.e.) of parameters (using the sandwich estimator; see McFadden and Train, 2000).

(3) For each data set $s$, compute the mean estimate for each model parameter across the draws. Label this as MED, and then take the mean of the MED values across the data sets to obtain a mean estimate. Compute the absolute percentage bias (APB) as: $A P B=\left|\frac{\text { mean estimate }- \text { true value }}{\text { true value }}\right| \times 100 .^{1}$

(4) For each data set $s$, compute the median s.e. for each model parameter across the 10 draws. Call this MSED, and then take the mean of the MSED values across the 20 data sets and label this as the asymptotic standard error (essentially this is the standard error of the distribution of the estimator as the sample size gets large). Note that we compute the median s.e. for each model parameter across the draws and label it as MSED rather than computing the mean s.e. for each model parameter across the draws. This is because, for some draws, the estimated standard errors turned out to be rather large relative to other independent standard error estimates for the same dataset. Note that the mean asymptotic standard error is a theoretical approximation to the finite sample standard error.

(5) Next, for each data set $s$, compute the simulation standard deviation for each parameter as the standard deviation in the estimated values across the independent draws (about the MED value). Call this standard deviation as SIMMED. For each parameter, take the mean of SIMMED across the different data sets. Label this as the simulation standard error for each parameter.

\footnotetext{
${ }^{1}$ In case a true parameter value is zero, the APB is computed by taking the difference of the mean estimate from the true value $(=0)$, dividing this difference by the value of 1 in the denominator, and multiplying by 100 .
} 
(6) For each parameter, compute a simulation adjusted asymptotic standard error as follows: $\sqrt{(\text { asymptotic standard error })^{2}+(\text { simulation standard error })^{2}}$

\section{MACML Approach}

(1) Estimate the MACML parameters for each data set $s$ and for each of 10 independent sets of permutations for computing the approximation for the likelihood function contribution of each individual. Obtain the time to get the convergent values (including the time to obtain the covariance matrix based on the inverse of the Godambe information matrix and the corresponding standard errors). Determine the mean time for convergence (TMACML) across the 200 estimation runs for each correlation pattern. As in the MSL runs, estimations were begun with the true values as the starting values, and the run times on different computers were scaled to an equivalent time on the baseline computer.

(2) For each data set $s$, estimate the standard errors (s.e.) (using the Godambe estimator; see Bhat, 2011a).

(3) For each data set $s$, compute the mean estimate for each model parameter across the 10 random permutations used. Label this as MED, and then take the mean of the MED values across the data sets to obtain a mean estimate. Compute the absolute percentage bias (APB) as in the MSL case.

(4) For each data set $s$, compute the median s.e. for each model parameter across the 10 draws. Call this MSED, and then take the mean of the MSED values across the 20 data sets and label this as the asymptotic standard error.

(5) Next, for each data set $s$, compute the approximation standard deviation for each parameter as the standard deviation in the estimated values across the independent permutations (about the MED value). Call this standard deviation as APPMED. For each parameter, take the mean of APPMED across the different data sets. Label this as the approximation standard error for each parameter.

(6) For each parameter, compute an approximation adjusted asymptotic standard error as follows: $\sqrt{(\text { asymptotic standard error })^{2}+{\text { (approximation standard error })^{2}}^{2}}$. 
The procedure above is applied for the cross-sectional random-coefficients case. For the panel inter-individual random coefficients case, and the panel inter-individual and intra-individual random coefficients case, the same approach as above is used, except that we generate only 10 datasets instead of 20 datasets. Also, only one MSL run is undertaken for each dataset, and so no simulation standard errors are computed for the MSL. This is because of the computational cost involved for each MSL run in the panel cases. However, we do compute the approximation standard errors for the MACML estimations by running 10 independent sets of permutations for each of the 10 datasets.

\subsection{Results}

\subsubsection{The Cross-Sectional Random Coefficients (CSRC) Model}

Table 1a presents the results for the CSRC model with a diagonal covariance matrix, and Table $1 \mathrm{~b}$ presents the corresponding results for the CSRC model with a non-diagonal covariance matrix.

\section{The Diagonal Case}

The results in Table 1a for the diagonal case indicate that both the MSL and the MACML method do reasonably well in recovering the parameters, as can be observed by comparing the mean estimate of the parameters with the true values (see the column titled "parameter estimates). The absolute percentage bias (APB) ranges from $7.3 \%$ to $13.3 \%$ (overall mean value of 9.8\% across parameters - see the row of the table labeled "Overall mean value across parameters" and the column titled "absolute percentage bias") for the MSL approach, and from $0.2 \%$ to $5.9 \%$ (overall mean value of $2.5 \%$ across parameters) for the MACML approach. Clearly, the MACML is able to recover parameters much more accurately than the MSL approach. For both the MSL and MACML methods, the APB values are generally somewhat smaller for the mean values of the distributions of the $\boldsymbol{\beta}$ parameter vector (i.e., the $b$ values in the table) than for the standard deviations of the distribution of the $\boldsymbol{\beta}$ parameter vector (i.e., the $\sigma$ parameters in the table). Also, there is more variation in the APB values among the $\sigma$ parameters than among the $b$ values, suggesting that the log-likelihood function is relatively flat for different values of standard deviations, leading to somewhat more difficulty in accurately recovering the standard deviation parameters. The sampling standard error values of the 
parameters indicate good efficiency of both the MSL and MACML estimators, with the asymptotic standard error being only about a tenth of the mean values of the estimator. The asymptotic standard error values may appear to suggest that the MSL estimator is marginally more efficient than the MACML estimator, given that the asymptotic standard errors from the MSL are slightly lower than from the MACML approach (the mean asymptotic standard error from the MSL method is 0.121, while the mean asymptotic standard error from the MACML method is 0.151). However, note that the lower standard errors from the MSL method are simply an artifact of the underestimation in recovering the true values of the parameters, which translates to consistently lower values of the mean parameter estimates from the MSL approach relative to the MACML method. In fact, in the MSL runs where the estimated parameters were of the order of the mean estimates from the MACML method, the corresponding MSL asymptotic standard errors were of the same order of magnitude as from the MACML method. Finally, the reader will note that the simulation standard error estimates are smaller than the sampling standard errors in the MSL approach, and similarly the approximation standard error estimates are smaller than the sampling standard errors in the MACML approach. On average, the simulation standard error is about $37 \%$ of the sampling standard error in the MSL case, while the approximation standard error is only about $13 \%$ of the sampling standard error in the MACML case. It is indeed quite remarkable that the approximation standard error with just a single permutation for approximating the likelihood function contribution of each individual in the MACML approach should be lower than the simulation standard error with 250 Halton draws per individual in the MSL approach. The final column provides the simulation-adjusted asymptotic standard error for the MSL case and the approximation-adjusted asymptotic standard error for the MACML case. These values are very close to the unadjusted asymptotic standard error in the MSL case and the unadjusted asymptotic standard error in the MACML case, once again indicating that the simulation and approximation errors are small relative to the sampling errors.

The time to convergence for the MSL estimation has a mean value of 66.1 minutes with a standard deviation of about 11 minutes. On the other hand, the time to convergence for the MACML estimation has a mean value of 1.96 minutes with a standard deviation of about 0.5 minutes. This indicates that the MACML method is about 33 times faster than the MSL estimation. Further, note that the MACML method is actually much more effective than 
suggested by this factor of 33, because it produces more accurate estimates than the MSL estimates. Some further explorations indicated that, even if the analyst increased the number of Halton draws to 450 per individual, the resulting APB (computed from 10 runs on 10 datasets of the 20 datasets) is in the order of $6.8 \%$ (relative to $2.5 \%$ for the MACML method), and the mean amount of time for convergence with 450 Halton draws is about 107 minutes, suggesting a time efficiency factor of well over 50 for the MACML method relative to the MSL method. This is indeed a phenomenal computational efficiency jump. As the number of random coefficients increase beyond five, one can only expect a further increase in the computational time advantage of the MACML over the MSL estimation approach. ${ }^{2}$

\section{The Non-Diagonal Case}

The results in Table $1 \mathrm{~b}$ provide information on the true mean values of the distribution of the $\boldsymbol{\beta}$ parameter vector (i.e., the $b$ values in the table) and the Cholesky-decomposed parameters characterizing the covariance matrix of the $\boldsymbol{\beta}$ parameter vector (i.e., the $l$ values in the table). The table also provides information on the mean estimates and the standard error estimates of the above parameters from the MSL and MACML approaches.

As in the diagonal case, the MSL and MACML methods perform well in terms of recovering the true parameter values. In fact, the MSL does marginally better than in the diagonal case, with the absolute percentage bias (APB) ranging from $0.5 \%$ to $22.9 \%$, with an overall mean APB value of 7.6\%. However, the MACML model still outperforms the MSL method, with an APB ranging from $0.6 \%$ to $17.8 \%$ and a mean APB value of $5.5 \%$. As in the diagonal case, there is more stability in the APB values across the mean values of the distribution of the $\boldsymbol{\beta}$ parameter vector (i.e., the $b$ values in the table) than for the Cholesky parameters characterizing the covariance matrix of the distribution of the $\boldsymbol{\beta}$ parameter vector (i.e., the $\boldsymbol{l}$ parameters in the table). The asymptotic standard error estimates again indicate good efficiency of both the MSL and MACML estimators, with the asymptotic standard error being only about a

\footnotetext{
${ }^{2}$ The mean asymptotic standard error with 450 draws turned out to be 0.136 compared to the corresponding value of 0.121 with 250 draws, reinforcing the observation earlier that the seemingly smaller asymptotic standard error from the MSL runs with 250 draws (compared to the MACML standard errors) is because of the underestimation in recovering the true values of the parameters, and the consistently lower values of the mean parameter estimates from the MSL approach relative to the MACML method. In the MSL estimation with 450 draws, the mean parameter estimates were closer to the true values (than with 250 draws), though still consistently lower than the mean parameter estimates from the MACML method.
} 
tenth of the mean values of the estimator for the $b$ values. Of course, the asymptotic standard errors for the $l$ parameters are a higher fraction of the mean estimates for these parameters, which is to be expected since many more parameters are being estimated in the covariance matrix. Between the MSL and the MACML estimators, the asymptotic standard errors are very similar in this non-diagonal case, with the mean standard error being 0.116 in the MSL case and 0.120 in the MACML case. This is because the MSL provides estimates that are closer to the true values, and to the values from the MACML estimation, unlike in the diagonal case. In terms of the simulation standard error in the MSL case and the approximation standard error in the MACML case, these are once again only a fraction of the sampling errors. However, as in the diagonal case, the simulation standard errors for the MSL case are much higher than the approximation standard errors from the MACML case. In particular, the simulation standard error is, on average, $49 \%$ of the sampling standard error in the MSL case, while the approximation error is, on average, only $12.5 \%$ of the sampling standard error in the MACML case.

The time to convergence for the MSL estimation has a mean value of 174.3 minutes (almost three hours) with a standard deviation of 28 minutes. In contrast, the time to convergence for the MACML estimation has a mean value of 5.20 minutes with a standard deviation of about 0.9 minutes. These results indicate that the MACML method is, once again and coincidentally, about 33 times faster than the MSL estimation with 250 Halton draws. However, for an applesto-apples comparison, one needs to improve the estimation with MSL, which we attempted to do by increasing the number of Halton draws. When using 450 draws per individual to estimate parameters using 10 runs on 10 datasets of the 20 datasets, the mean APB value turned out to be 9.6\% with a mean time of convergence of about 380 minutes. This mean APB is higher compared to the 250 draws per individual case, and raises a yet unexplored issue with the Halton and related QMC draws. That is, the effectiveness of the standard and/or scrambled QMC draws may not be stable as the number of draws is increased, because the cycling of the QMC sequences may lead to poor coverage of the multivariate space for any given individual for specific numbers of draws.

\subsubsection{Panel Inter-Individual Random Coefficients (PIRC) model}

The panel estimations are undertaken with about half the number of total observations as the cross-sectional estimations, so that the computation time can be kept to a reasonable time with 
the MSL approach. Further, the covariance matrix for the PIRC model is estimated based on individual-level random heterogeneity, based on 500 distinct draws (one for each of the 500 individuals). Also, to keep the computation time reasonable, we use only 10 datasets in the panel case. As a result, one may expect the level of performance of the MACML and the MSL to be, in general, somewhat lower than the cross-sectional case. In the next two sections, we discuss the results for the diagonal and non-diagonal cases.

\section{The Diagonal Case}

The results in Table 2a for the diagonal case indicate that, for the MSL estimation approach, the absolute percentage bias (APB) ranges from 16.0\% to 19.9\%, with an overall mean APB of 17.1\%. The corresponding APB values for the MACML approach range from $5.8 \%$ to $12.4 \%$, with an overall mean value of $8.0 \%$. As in the cross-sectional case, the MSL estimation is undertaken with 250 scrambled and randomized Halton draws, while the MACML estimation is undertaken with a single randomized permutation (except in 6 of the 100 cases, where two randomized permutations provided stability). ${ }^{3}$ Clearly, the MACML is able to recover parameters more accurately than the MSL approach. The seemingly lower asymptotic standard errors of the MSL approach is again an artifact of the substantial underestimation of parameter values in the MSL approach. The approximation standard error estimates are smaller than the sampling standard errors in the MACML approach. On average, the approximation standard error is only about $28 \%$ of the sampling standard error in the MACML case. ${ }^{4}$

The time to convergence for the MSL estimation has a mean value of 96.3 minutes with a standard deviation of about 11 minutes. On the other hand, the time to convergence for the MACML estimation has a mean value of 12.4 minutes with a standard deviation of about 3 minutes. Compared to the cross-section case, the computational efficiency of the MACML over the MSL is not as substantial in the panel diagonal case. This is because of two reasons. The first is that the number of multivariate integrations per likelihood function or gradient iteration is only 500 in the panel case (corresponding to the 500 individuals), and this benefits the MSL approach.

\footnotetext{
${ }^{3}$ All time computations discussed later for the one-permutation case include the times for these 6 cases that used two permutations per individual.

${ }^{4}$ When we estimated the panel diagonal case with two permutations per individual (rather than one permutation per individual), the mean APB improved marginally to 7.0\%. The approximation standard error, on average, turned out to be $15 \%$ of the asymptotic sampling standard error in this case. Overall, the results show the ability to recover parameters with small approximation error with just one permutation per individual.
} 
Second, in the MACML estimation of the panel case, we consider all the ten pairings of the 5 choice occasions per individual, which increases the number of multi-dimensional integrals to be evaluated using the approximation method to 5000 (500 individuals times 10 pairings per individual). However, the MACML approach still retains a significant computation edge, being about 8 times faster than the MSL approach with 250 randomized and scrambled Halton draws. At the same time, the MACML approach is able to recover parameters much more accurately than the MSL approach. In fact, even when the number of Halton draws was increased to 450 per individual, the MSL had a mean $\mathrm{APB}$ of $14.3 \%$, and the corresponding mean time of convergence was 185.6 minutes. This indicates that the actual computation edge of the MACML over the MSL is more than 15 -fold. Note also that as soon as slightly more complicated (and more realistic) structures such as autoregressive random coefficients over choice occasions, or both choice occasion-specific and individual-specific random coefficients, or both individualspecific and across-individual random coefficients get introduced in the model, the MSL

becomes extremely time consuming and close to being infeasible to estimate (as we will show in Section 3.2.3). One other problem we found even in this simple panel MSL estimation was that one of the ten runs experienced non-convergence problems. On the other hand, no convergence issues were encountered with the MACML estimation.

\section{The Non-Diagonal Case}

The results for the panel non-diagonal random coefficients case are provided in Table $2 \mathrm{~b}$. As can be observed, the average APB is somewhat higher in this case relative to the diagonal case, mainly due to the APBs associated with the Cholesky parameters that determine the full covariance matrix. However, the high APB is somewhat deceiving, because the estimated values of the Cholesky parameters are not too far away from the true values. But the small values of the true Cholesky parameter values tend to inflate the APB values. Also, as indicated earlier, with a limited sample size and several parameters to estimate, this is not an unexpected result. The main point to note is that the MACML continues to do a much better job in recovering parameters than the MSL, and with at least an 8-fold or so reduction in computational cost. The actual computational efficiency is much higher, but we did not go beyond 250 Halton draws in the MSL case as in the earlier cases because of the very high computation costs involved. As importantly, we did notice an increase in convergence problems with the MSL approach in the 
non-diagonal case, with 5 of the 10 runs getting bogged down and not going anywhere. There were no convergence issues whatsoever with the MACML approach.

\subsubsection{Panel Intra-Individual and Inter-Individual Random Coefficients}

The MSL estimation of the situation when there are both intra-individual and inter-individual random coefficients is extremely expensive from a computational standpoint, since there are two levels of random coefficients to be integrated out (see Equations (5) and (6)). This implies that if $Q$ draws of a QMC sequence were to be used for each level of integration, then for each of the $Q$ draws of the outer integral, the inner integral itself will need to be evaluated using $Q$ draws. Thus, the number of total draws becomes $Q^{2}$. If we are to use 250 draws as we have done in the cross-sectional and panel cases earlier, the total number of draws would be 62,500. Effectively, it is practically infeasible to estimate such a model accurately using the MSL technique within a reasonable amount of time. However, the model does not pose any problems for estimation using the MACML approach; the computational cost is about the same order as for the simple panel case or the simple cross-sectional case. In this section, we provide only the results for the MACML estimation. The MSL estimation with 250 draws for each integration level was taking about 3 hours per iteration. Assuming convergence in 55 iterations (which was about the average for the case for the pure panel diagonal random coefficients case), the time for the MSL estimation would be about 165 hours or about 7 days for the diagonal covariance specification. This is in contrast to about 25 minutes for the MACML estimation of the diagonal case, and 50 minutes for the MACML estimation of the non-diagonal case.

\section{The Diagonal Case}

The results for the diagonal case are presented in Table 3a. The mean APB in this case is $12.6 \%$, with the individual parameter APBs varying from $8.1 \%$ to $18.5 \%$. The APB is affected here by the number of covariance-related parameters to be estimated, as also reflected in the higher asymptotic standard errors of the parameters compared to the previous cases. But it is indeed remarkable that the MACML method does about as well as for the panel inter-individual diagonal case of Section 3.2.2, both in terms of recovering parameters as well as computation time. Specifically, in terms of computation time, the model takes, on average over the 100 runs (10 different runs on 10 different data samples), only about 23 minutes for convergence, which is 
about twice the amount of time as for the panel inter-individual case. The standard deviation of the times for convergence over the 100 runs is about 4.5 minutes. At the same time, the approximation error remains very small, at an average of about $18 \%$ of the asymptotic standard error. Of course, the ability to recover parameters may be improved by increasing the number of permutations per individual used in the MACML estimation. In our experiments, the APB reduced from $12.6 \%$ to $10.8 \%$, when the number of permutations was increased to 2 , and further reduced to $7.7 \%$ when the number of permutations was increased to 5 . However, the mean time to convergence increased from 23 minutes (for one permutation per individual) to 44 minutes (for two permutations per individual) to 117 minutes (for five permutations per individual).

\section{The Non-Diagonal Case}

This estimation involves the most number of parameters, including five mean parameters on the five independent variables, 16 covariance elements from the individual-specific covariance matrix, and another 16 covariance elements from the choice occasion-specific covariance matrix (see Table 3b). The mean APB here is about $15.3 \%$, though the APB values for the mean parameters are recovered very accurately (the APB values for the mean parameters range from $3.7 \%$ to $6.8 \%$, which is even better than the corresponding APB values for the diagonal case). The relatively high APB values for the Cholesky parameters are a result of the high number of such parameters estimated from a sample size of 500 individuals and 2500 choice occasions, as well as the small magnitudes of the true values of the Cholesky parameters. When the number of permutations per individual was increased to two, the mean APB reduced to $14.1 \%$. The mean APB further reduced marginally to $13.1 \%$ with five permutations per individual. The time to convergence with one permutation per individual is about twice the time needed for the diagonal case with one permutation per individual, but is still only of the order of 46 minutes on average. The times to convergence for two and five permutations per individual are also about twice the corresponding times in the diagonal case.

\section{SUMMARY AND CONCLUSIONS}

Random coefficients discrete choice models are increasingly being used for unordered response multinomial choice modeling in the transportation and other fields, as a means to accommodate varying tastes across decision makers due to unobserved (to the analyst) factors. In such random 
coefficients models, the overall error term vector is effectively decomposed into an independent and identically distributed (IID) component vector and another non-IID (across alternatives) vector of jointly distributed random coefficients. While various different distributions may be used for the non-IID component, it is common practice to employ a multivariate normal distribution. If the analyst uses a normal distribution for the IID portion, the result is the "mixed" multinomial probit (MMNP) model.

The MMNP model structure may be applied to both cross-sectional and panel contexts. In either case, both the model structures do not have an analytically tractable form for the choice probabilities and for the likelihood function. The approach used to estimate such models is typically based on pseudo-Monte Carlo or quasi-Monte Carlo simulation techniques to evaluate the multidimensional integrals in these models. In such an MSL estimation approach, consistency, efficiency, and asymptotic normality of the estimator is critically predicated on the condition that the number of simulation draws per individual rises faster than the square root of the number of individuals in the estimation sample. This effectively implies that the desirable asymptotic properties of the MSL estimator are obtained at the expense of computational cost. Also, the simulation noise when dealing with high dimensionalities of integration can cause convergence problems.

Bhat (2011a) recently proposed a maximum approximated composite marginal likelihood (MACML) method for the estimation of MNP-based models. In this current paper, the focus is on evaluating the ability of the MACML method to recover parameters of MMNP models from finite samples, and to compare the performance of the MACML estimator with the MSL estimator in terms of finite sample bias in parameters and the computational time for estimation. Within the class of MMNP models, we examine three different model structures: the crosssectional random coefficients structure, the panel inter-individual random coefficients structure, and the panel intra- and inter-individual random coefficients structure. Within each of these structures, both the cases of independent random coefficients (i.e., the diagonal covariance specification) and dependent random coefficients (i.e., the non-diagonal covariance specification) are considered.

The results of the analysis indicate that the MACML recovers parameters much more accurately than the MSL approach in all model structures and covariance specifications. The MACML inference approach also estimates the parameters efficiently, with the asymptotic 
standard errors being, in general, only a small proportion of the true values. It is remarkable that the approximation error involved in the use of even only a single permutation to evaluate the MVNCD function is very small, being only about $26-42 \%$ of the simulation error associated with 250 randomized and scrambled Halton draws in the cross-sectional model estimations (the simulation errors were not estimated in the MSL approach for the panel cases because of the computational costs involved in running multiple runs on the same data set). As importantly, the MACML inference approach takes only a small fraction of the time needed for MSL estimation. In particular, the results suggest that the MACML approach is about 50 times faster than the MSL for the cross-sectional random coefficients case, at least 15 times faster than the MSL for the panel inter-individual random coefficients case, and about 350 times or more faster than the MSL for the panel intra- and inter-individual random coefficients case. As the number of alternatives in the unordered-response model increases, one can expect even higher computational efficiency factors for the MACML over the MSL approach. Further, as evident in the panel intra- and inter-individual random coefficients case, the MSL is all but practically infeasible when the mixing structure leads to an explosion in the dimensionality of integration in the likelihood function, but these situations are handled with ease in the MACML approach.

Of course, the results above are specific to the mixed multinomial probit (MMNP) structure. If one insists on using the mixed multinomial logit structure (MMNL) structure, the MACML method needs to be supplemented with a normal scale mixture technique to approximate the IID extreme value error distribution. Bhat (2011b) provides the procedure. Comparisons of the MACML and MSL estimations for the MMNL model structure would be of interest for the cross-sectional and inter-individual panel cases, though the MACML procedure should continue to provide substantial computational benefits in the more involved panel intraand inter-individual case. In any case, the movement between the use of the MMNL and the MMNP structures has been dictated primarily by ease of estimation. In the past several years, the scale has been tilted more toward the use of the MMNL structure, primarily because of the ease of conceptualization and coding of the simulation procedure for the MMNL structure (see Bhat et al., 2008 and Train, 2009). However, in the MACML estimation technique, the MMNP model is easier to estimate because of the conjugate property of addition of the normal distribution. Thus, we may expect to see the scale tilting back toward the MMNP structure for the specific case when the mixing distribution is continuous and normal. When the mixing distribution is not 
normal, one can still use the MACML approach by approximating the continuous multivariate distribution using a multivariate normal scale mixture, but this will increase computational cost in proportion to the number of dimensions in the mixing distribution. In such cases, the MSL inference approach may continue to be the choice method for estimation of the simple models, though the MACML supplemented by the scale mixture technique would perhaps still be a promising way to proceed for more complicated models (such as the case with panel intra- and inter-individual random coefficients).

The current paper also generates several research issues for the future. First, it would be useful to undertake a similar analysis as the one here for varying numbers of random coefficients (say, 10 and 20 random coefficients) to examine the effectiveness of the MACML approach with different numbers of random coefficients. ${ }^{5}$ Conceptually, this should not have much of an effect on the MACML procedure, but the empirical evidence needs to be generated. Second, the MACML likelihood procedure and the gradient procedure have been coded in the GAUSS matrix programming language. Currently, a scalar version of the MVNCD approximation procedure has been coded, which implies that the MACML code calls the MVNCD code each time a MVNCD function is to be approximated. This MACML approach can be speeded up by vectorizing the MVNCD approximation procedure, so that the procedure returns the approximated values for multiple MVNCD evaluations at once. Third, the MVNCD procedure is written to cycle through until a permutation is used that provides a non-negative value for the MVNCD function evaluation. That is, it is possible that the first permutation leads to a value for the MVNCD function approximation that is non-negative, in which case the code automatically attempts a different permutation for the decomposition of the MVNCD function into marginal and conditional probabilities. This situation is relatively rare, but can happen as the gradient procedure searches for an update direction. In such situations, one may seek a different permutation (as we have done) or just increase the number of permutations to make the approximation. These, and potentially other automated techniques, can be compared in future research. Finally, it is hoped that the MACML procedure will spawn empirical research into behaviorally rich model specifications within the context of unordered multinomial choice

\footnotetext{
${ }^{5}$ Of course, one has to be careful when increasing the number of correlated random coefficients, since this can lead to identification fragility.
} 
modeling, including autoregressive random coefficients, dynamics in coefficients, space-time effects, and spatial/social interactions.

In closing, the MACML inference approach has the potential to dramatically influence the use of the mixed multinomial probit model in practice, and should facilitate the practical application of rich model structures for unordered-response discrete choice modeling.

\section{ACKNOWLEDGMENTS}

The authors acknowledge the helpful comments of two anonymous reviewers on an earlier version of the paper. Naveen Eluru assisted with the maximum simulated likelihood (MSL) codes. Finally, the authors are grateful to Lisa Macias for her help in typesetting and formatting this document. 


\section{REFERENCES}

Bhat, C.R., 2003. Simulation estimation of mixed discrete choice models using randomized and scrambled Halton sequences. Transportation Research Part B 37(9), 837-855.

Bhat, C.R., 2011a. The maximum approximated composite marginal likelihood (MACML) estimation of multinomial probit-based unordered response choice models. Technical paper, Department of Civil, Architectural and Environmental Engineering, The University of Texas at Austin.

http://www.caee.utexas.edu/prof/bhat/ABSTRACTS/MACML_unordered_response_paper.pdf.

Bhat, C.R., 2011b. The MACML estimation of the normally-mixed multinomial logit model. Technical paper, Department of Civil, Architectural and Environmental Engineering, The University of Texas at Austin. http://www.caee.utexas.edu/prof/bhat/ABSTRACTS/MACML_Estim_Norm_MML_Model.pdf

Bhat, C.R., Castelar, S., 2002. A unified mixed logit framework for modeling revealed and stated preferences: formulation and application to congestion pricing analysis in the San Francisco Bay area. Transportation Research Part B 36(7), 593-616.

Bhat, C.R., Sardesai, R., 2006. The impact of stop-making and travel time reliability on commute mode choice. Transportation Research Part B 40(9), 709-730.

Bhat, C.R., Eluru, N., Copperman, R.B., 2008. Flexible model structures for discrete choice analysis. In Handbook of Transport Modelling, 2nd edition, Hensher, D.A., Button, K.J., (eds.), Elsevier Science, 75-104.

Hess, S., Rose, J.M., 2009. Allowing for intra-respondent variations in coefficients estimated on repeated choice data. Transportation Research Part B 43(6), 708-719.

McFadden, D., Train, K., 2000. Mixed MNL models for discrete response. Journal of Applied Econometrics 15(5), 447-470.

Sandor, Z., Train, K., 2004. Quasi-random simulation of discrete choice models. Transportation Research Part B 38(4), 313-327.

Sivakumar, A., Bhat, C.R., Ökten, G., 2005. Simulation estimation of mixed discrete choice models with the use of randomized quasi-Monte Carlo sequences: a comparative study. Transportation Research Record 1921, 112-122.

Train, K. 2009. Discrete Choice Methods with Simulation, $2^{\text {nd }}$ ed., Cambridge University Press, Cambridge. 


\section{LIST OF TABLES}

Table 1a: Evaluation of the ability to recover true parameters for the cross-sectional diagonal case

Table 1b: Evaluation of the ability to recover true parameters for the cross-sectional nondiagonal case

Table 2a: Evaluation of the ability to recover true parameters for the panel inter-individual random coefficients diagonal case

Table 2b: Evaluation of the ability to recover true parameters for the panel inter-individual random coefficients non-diagonal case

Table 3a: Evaluation of the ability to recover true parameters for the panel intra-individual and inter-individual random coefficients diagonal case

Table 3b: Evaluation of the ability to recover true parameters for the panel intra-individual and inter-individual random coefficients non-diagonal case 
Table 1a: Evaluation of the ability to recover true parameters for the cross-sectional diagonal case

\begin{tabular}{|c|c|c|c|c|c|c|c|c|c|c|c|}
\hline \multirow[b]{3}{*}{ Parameter } & \multirow[b]{3}{*}{$\begin{array}{l}\text { True } \\
\text { Value }\end{array}$} & \multicolumn{5}{|c|}{ MSL Method } & \multicolumn{5}{|c|}{ MACML Method } \\
\hline & & \multicolumn{2}{|c|}{ Parameter Estimates } & \multicolumn{3}{|c|}{ Standard Error Estimates } & \multicolumn{2}{|c|}{ Parameter Estimates } & \multicolumn{3}{|c|}{ Standard Error Estimates } \\
\hline & & $\begin{array}{c}\text { Mean } \\
\text { Estimate }\end{array}$ & $\begin{array}{c}\text { Absolute } \\
\text { Percentage } \\
\text { Bias }\end{array}$ & $\begin{array}{l}\text { Asymptotic } \\
\text { Standard } \\
\text { Error }\end{array}$ & $\begin{array}{l}\text { Simulation } \\
\text { Standard } \\
\text { Error }\end{array}$ & $\begin{array}{l}\text { Simulation } \\
\text { Adjusted } \\
\text { Asymptotic } \\
\text { Standard } \\
\text { Error } \\
\end{array}$ & $\begin{array}{c}\text { Mean } \\
\text { Estimate }\end{array}$ & $\begin{array}{c}\text { Absolute } \\
\text { Percentage } \\
\text { Bias }\end{array}$ & $\begin{array}{l}\text { Asymptotic } \\
\text { Standard } \\
\text { Error }\end{array}$ & $\begin{array}{l}\text { Approximation } \\
\text { Standard } \\
\text { Error }\end{array}$ & $\begin{array}{c}\text { Approximation } \\
\text { Adjusted } \\
\text { Asymptotic } \\
\text { Standard } \\
\text { Error } \\
\end{array}$ \\
\hline \multicolumn{12}{|c|}{ Mean values of the $\beta$ vector } \\
\hline$b 1$ & 1.500 & 1.366 & $9.0 \%$ & 0.129 & 0.050 & 0.139 & 1.472 & $1.9 \%$ & 0.167 & 0.022 & 0.169 \\
\hline$b 2$ & -1.000 & -0.906 & $9.4 \%$ & 0.089 & 0.033 & 0.095 & -0.976 & $2.4 \%$ & 0.113 & 0.014 & 0.114 \\
\hline$b 3$ & 2.000 & 1.801 & $10.0 \%$ & 0.167 & 0.066 & 0.180 & 1.940 & $3.0 \%$ & 0.218 & 0.028 & 0.219 \\
\hline$b 4$ & 1.000 & 0.906 & $9.4 \%$ & 0.089 & 0.034 & 0.095 & 0.977 & $2.3 \%$ & 0.114 & 0.014 & 0.114 \\
\hline$b 5$ & -2.000 & -1.820 & $9.0 \%$ & 0.170 & 0.067 & 0.182 & -1.960 & $2.0 \%$ & 0.220 & 0.028 & 0.222 \\
\hline \multicolumn{12}{|c|}{ Standard deviations of the $\beta$ vector } \\
\hline$\sigma 2$ & 1.000 & 0.906 & $9.4 \%$ & 0.111 & 0.040 & 0.118 & 0.984 & $1.6 \%$ & 0.136 & 0.016 & 0.137 \\
\hline$\sigma 3$ & 1.000 & 0.867 & $13.3 \%$ & 0.112 & 0.041 & 0.119 & 0.941 & $5.9 \%$ & 0.135 & 0.017 & 0.136 \\
\hline$\sigma 4$ & 1.000 & 0.904 & $9.6 \%$ & 0.111 & 0.040 & 0.118 & 0.982 & $1.8 \%$ & 0.136 & 0.017 & 0.137 \\
\hline$\sigma 5$ & 1.000 & 0.927 & $7.3 \%$ & 0.117 & 0.041 & 0.124 & 1.002 & $0.2 \%$ & 0.140 & 0.016 & 0.141 \\
\hline \multicolumn{2}{|c|}{$\begin{array}{l}\text { Overall Mean Value } \\
\text { Across Parameters } \\
\end{array}$} & - & $9.8 \%$ & 0.121 & 0.045 & 0.129 & - & $2.5 \%$ & 0.151 & 0.019 & 0.153 \\
\hline \multicolumn{2}{|c|}{ Mean Time } & \multicolumn{5}{|c|}{66.09} & \multicolumn{5}{|c|}{1.96} \\
\hline \multicolumn{2}{|c|}{ Std. dev of Time } & \multicolumn{5}{|c|}{10.87} & \multicolumn{5}{|c|}{0.42} \\
\hline \multicolumn{2}{|c|}{$\%$ of Runs Converged } & \multicolumn{5}{|c|}{$100 \%$} & \multicolumn{5}{|c|}{$100 \%$} \\
\hline
\end{tabular}


Table 1b: Evaluation of the ability to recover true parameters for the cross-sectional non-diagonal case

\begin{tabular}{|c|c|c|c|c|c|c|c|c|c|c|c|}
\hline \multirow[b]{3}{*}{ Parameter } & \multirow[b]{3}{*}{$\begin{array}{l}\text { True } \\
\text { Value }\end{array}$} & \multicolumn{5}{|c|}{ MSL Method } & \multicolumn{5}{|c|}{ MACML Method } \\
\hline & & \multicolumn{2}{|c|}{ Parameter Estimates } & \multicolumn{3}{|c|}{ Standard Error Estimates } & \multicolumn{2}{|c|}{ Parameter Estimates } & \multicolumn{3}{|c|}{$\begin{array}{c}\text { Standard Error Estimates } \\
\end{array}$} \\
\hline & & $\begin{array}{c}\text { Mean } \\
\text { Estimate }\end{array}$ & $\begin{array}{c}\text { Absolute } \\
\text { Percentage } \\
\text { Bias }\end{array}$ & $\begin{array}{l}\text { Asymptotic } \\
\text { Standard } \\
\text { Error }\end{array}$ & $\begin{array}{c}\text { Simulation } \\
\text { Standard } \\
\text { Error }\end{array}$ & $\begin{array}{l}\text { Simulation } \\
\text { Adjusted } \\
\text { Asymptotic } \\
\text { Standard } \\
\text { Error } \\
\end{array}$ & $\begin{array}{c}\text { Mean } \\
\text { Estimate }\end{array}$ & $\begin{array}{c}\text { Absolute } \\
\text { Percentage } \\
\text { Bias }\end{array}$ & $\begin{array}{l}\text { Asymptotic } \\
\text { Standard } \\
\text { Error }\end{array}$ & $\begin{array}{l}\text { Approximation } \\
\text { Standard } \\
\text { Error }\end{array}$ & $\begin{array}{c}\text { Approximation } \\
\text { Adjusted } \\
\text { Asymptotic } \\
\text { Standard } \\
\text { Error } \\
\end{array}$ \\
\hline \multicolumn{12}{|c|}{ Mean values of the $\beta$ vector } \\
\hline$b 1$ & 1.500 & 1.374 & $8.4 \%$ & 0.133 & 0.049 & 0.142 & 1.443 & $3.8 \%$ & 0.147 & 0.022 & 0.148 \\
\hline$b 2$ & -1.000 & -0.912 & $8.8 \%$ & 0.093 & 0.037 & 0.100 & -0.959 & $4.1 \%$ & 0.102 & 0.014 & 0.103 \\
\hline$b 3$ & 2.000 & 1.830 & $8.5 \%$ & 0.174 & 0.068 & 0.187 & 1.923 & $3.8 \%$ & 0.191 & 0.029 & 0.193 \\
\hline$b 4$ & 1.000 & 0.914 & $8.6 \%$ & 0.092 & 0.032 & 0.097 & 0.958 & $4.2 \%$ & 0.101 & 0.014 & 0.102 \\
\hline$b 5$ & -2.000 & -1.849 & $7.6 \%$ & 0.176 & 0.068 & 0.189 & -1.941 & $3.0 \%$ & 0.194 & 0.028 & 0.196 \\
\hline \multicolumn{12}{|c|}{ Cholesky parameters characterizing the covariance matrix of the $\beta$ vector } \\
\hline 111 & 1.000 & 0.909 & $9.1 \%$ & 0.112 & 0.040 & 0.119 & 0.959 & $4.1 \%$ & 0.119 & 0.017 & 0.120 \\
\hline 112 & -0.500 & -0.463 & $7.3 \%$ & 0.085 & 0.029 & 0.090 & -0.472 & $5.6 \%$ & 0.085 & 0.010 & 0.085 \\
\hline$l 14$ & 0.750 & 0.689 & $8.2 \%$ & 0.092 & 0.028 & 0.097 & 0.707 & $5.7 \%$ & 0.095 & 0.013 & 0.096 \\
\hline$l 15$ & 0.000 & 0.006 & $0.6 \%$ & 0.086 & 0.040 & 0.095 & 0.015 & $1.5 \%$ & 0.088 & 0.008 & 0.089 \\
\hline 122 & 0.866 & 0.756 & $12.7 \%$ & 0.109 & 0.043 & 0.117 & 0.809 & $6.5 \%$ & 0.116 & 0.017 & 0.117 \\
\hline 123 & 0.433 & 0.431 & $0.5 \%$ & 0.105 & 0.050 & 0.117 & 0.436 & $0.6 \%$ & 0.100 & 0.012 & 0.101 \\
\hline 124 & -0.144 & -0.149 & $3.6 \%$ & 0.101 & 0.041 & 0.109 & -0.170 & $17.8 \%$ & 0.093 & 0.010 & 0.094 \\
\hline 125 & 0.000 & -0.021 & $2.1 \%$ & 0.101 & 0.055 & 0.115 & -0.019 & $1.9 \%$ & 0.098 & 0.010 & 0.099 \\
\hline 133 & 0.866 & 0.750 & $13.4 \%$ & 0.130 & 0.073 & 0.149 & 0.812 & $6.3 \%$ & 0.131 & 0.019 & 0.132 \\
\hline 134 & 0.237 & 0.242 & $2.0 \%$ & 0.112 & 0.055 & 0.125 & 0.259 & $9.3 \%$ & 0.106 & 0.011 & 0.106 \\
\hline 135 & 0.000 & -0.031 & $3.1 \%$ & 0.120 & 0.081 & 0.145 & -0.029 & $2.9 \%$ & 0.116 & 0.011 & 0.117 \\
\hline 144 & 0.601 & 0.464 & $22.9 \%$ & 0.126 & 0.085 & 0.152 & 0.531 & $11.6 \%$ & 0.125 & 0.015 & 0.126 \\
\hline 145 & 0.000 & -0.053 & $5.3 \%$ & 0.168 & 0.134 & 0.214 & -0.053 & $5.3 \%$ & 0.171 & 0.017 & 0.172 \\
\hline 155 & 1.000 & 0.885 & $11.5 \%$ & 0.125 & 0.089 & 0.153 & 0.956 & $4.4 \%$ & 0.136 & 0.018 & 0.137 \\
\hline \multicolumn{2}{|c|}{$\begin{array}{l}\text { Overall Mean Value } \\
\text { Across Parameters }\end{array}$} & - & $7.6 \%$ & 0.116 & 0.057 & 0.130 & - & $5.5 \%$ & 0.120 & 0.015 & 0.121 \\
\hline \multicolumn{2}{|c|}{ Mean Time } & \multicolumn{5}{|c|}{1744.32} & \multicolumn{5}{|c|}{5.19} \\
\hline
\end{tabular}


Table 2a: Evaluation of the ability to recover true parameters for the panel inter-individual random coefficients diagonal case

\begin{tabular}{|c|c|c|c|c|c|c|c|c|c|}
\hline \multirow{3}{*}{ Parameter } & \multirow{3}{*}{$\begin{array}{l}\text { True } \\
\text { Value }\end{array}$} & \multicolumn{3}{|c|}{ MSL Method } & \multicolumn{5}{|c|}{ MACML Method } \\
\hline & & \multicolumn{2}{|c|}{ Parameter Estimates } & \multirow{2}{*}{\begin{tabular}{|c|}
$\begin{array}{c}\text { Standard Error } \\
\text { Estimates }\end{array}$ \\
\\
Asymptotic \\
Standard Error
\end{tabular}} & \multicolumn{2}{|c|}{ Parameter Estimates } & \multicolumn{3}{|c|}{ Standard Error Estimates } \\
\hline & & $\begin{array}{c}\text { Mean } \\
\text { Estimate }\end{array}$ & $\begin{array}{c}\text { Absolute } \\
\text { Percentage } \\
\text { Bias }\end{array}$ & & $\begin{array}{c}\text { Mean } \\
\text { Estimate }\end{array}$ & $\begin{array}{c}\text { Absolute } \\
\text { Percentage } \\
\text { Bias }\end{array}$ & $\begin{array}{l}\text { Asymptotic } \\
\text { Standard } \\
\text { Error }\end{array}$ & $\begin{array}{l}\text { Approximation } \\
\text { Standard } \\
\text { Error }\end{array}$ & $\begin{array}{c}\text { Approximation } \\
\text { Adjusted } \\
\text { Asymptotic } \\
\text { Standard Error } \\
\end{array}$ \\
\hline \multicolumn{10}{|c|}{ Mean values of the $\beta$ vector } \\
\hline$b 1$ & 1.500 & 1.247 & $16.9 \%$ & 0.094 & 1.400 & $6.6 \%$ & 0.143 & 0.043 & 0.149 \\
\hline$b 2$ & -1.000 & -0.818 & $18.2 \%$ & 0.070 & -0.914 & $8.6 \%$ & 0.102 & 0.028 & 0.106 \\
\hline$b 3$ & 2.000 & 1.660 & $17.0 \%$ & 0.111 & 1.869 & $6.6 \%$ & 0.185 & 0.056 & 0.194 \\
\hline$b 4$ & 1.000 & 0.840 & $16.0 \%$ & 0.075 & 0.935 & $6.5 \%$ & 0.106 & 0.029 & 0.110 \\
\hline$b 5$ & -2.000 & -1.670 & $16.5 \%$ & 0.111 & -1.870 & $6.5 \%$ & 0.184 & 0.056 & 0.193 \\
\hline \multicolumn{10}{|c|}{ Standard deviations of the $\beta$ vector } \\
\hline$\sigma 1$ & 1.000 & 0.834 & $16.6 \%$ & 0.086 & 0.942 & $5.8 \%$ & 0.120 & 0.034 & 0.124 \\
\hline$\sigma 2$ & 1.000 & 0.801 & $19.9 \%$ & 0.077 & 0.876 & $12.4 \%$ & 0.111 & 0.031 & 0.116 \\
\hline$\sigma 3$ & 1.000 & 0.844 & $15.6 \%$ & 0.093 & 0.910 & $9.0 \%$ & 0.121 & 0.032 & 0.125 \\
\hline$\sigma 4$ & 1.000 & 0.821 & $17.9 \%$ & 0.084 & 0.921 & $7.9 \%$ & 0.119 & 0.031 & 0.123 \\
\hline$\sigma 5$ & 1.000 & 0.836 & $16.4 \%$ & 0.083 & 0.900 & $10.0 \%$ & 0.119 & 0.033 & 0.124 \\
\hline \multicolumn{2}{|c|}{$\begin{array}{l}\text { Overall Mean Value } \\
\text { Across Parameters }\end{array}$} & - & $17.1 \%$ & 0.088 & - & $8.0 \%$ & 0.131 & 0.037 & 0.136 \\
\hline \multicolumn{2}{|c|}{ Mean Time } & \multicolumn{3}{|c|}{96.26} & \multicolumn{5}{|c|}{12.35} \\
\hline \multicolumn{2}{|c|}{ Std. dev of Time } & \multicolumn{3}{|c|}{11.13} & \multicolumn{5}{|c|}{3.01} \\
\hline \multicolumn{2}{|c|}{ \% of Runs Converged } & \multicolumn{3}{|c|}{$90 \%$} & \multicolumn{5}{|c|}{$100 \%$} \\
\hline
\end{tabular}


Table 2b: Evaluation of the ability to recover true parameters for the panel inter-individual random coefficients non-diagonal case

\begin{tabular}{|c|c|c|c|c|c|c|c|c|c|}
\hline \multirow[b]{3}{*}{ Parameter } & \multirow{3}{*}{$\begin{array}{l}\text { True } \\
\text { Value }\end{array}$} & \multicolumn{3}{|c|}{ MSL Method } & \multicolumn{5}{|c|}{ MACML Method } \\
\hline & & \multicolumn{2}{|c|}{ Parameter Estimates } & \multirow{2}{*}{$\begin{array}{c}\begin{array}{c}\text { Standard Error } \\
\text { Estimates }\end{array} \\
\\
\text { Asymptotic } \\
\text { Standard Error }\end{array}$} & \multicolumn{2}{|c|}{ Parameter Estimates } & \multicolumn{3}{|c|}{ Standard Error Estimates } \\
\hline & & $\begin{array}{c}\text { Mean } \\
\text { Estimate }\end{array}$ & $\begin{array}{c}\text { Absolute } \\
\text { Percentage } \\
\text { Bias }\end{array}$ & & $\begin{array}{c}\text { Mean } \\
\text { Estimate }\end{array}$ & $\begin{array}{c}\text { Absolute } \\
\text { Percentage } \\
\text { Bias }\end{array}$ & $\begin{array}{l}\text { Asymptotic } \\
\text { Standard } \\
\text { Error }\end{array}$ & $\begin{array}{c}\text { Approximation } \\
\text { Standard } \\
\text { Error }\end{array}$ & $\begin{array}{c}\text { Approximation } \\
\text { Adjusted } \\
\text { Asymptotic } \\
\text { Standard Error }\end{array}$ \\
\hline \multicolumn{10}{|c|}{ Mean values of the $\beta$ vector } \\
\hline$b 1$ & 1.500 & 1.296 & $13.6 \%$ & 0.103 & 1.394 & $7.1 \%$ & 0.133 & 0.034 & 0.138 \\
\hline$b 2$ & -1.000 & -0.866 & $13.4 \%$ & 0.076 & -0.943 & $5.7 \%$ & 0.099 & 0.025 & 0.102 \\
\hline$b 3$ & 2.000 & 1.747 & $12.7 \%$ & 0.133 & 1.879 & $6.1 \%$ & 0.171 & 0.046 & 0.177 \\
\hline$b 4$ & 1.000 & 0.850 & $15.0 \%$ & 0.078 & 0.920 & $8.0 \%$ & 0.098 & 0.023 & 0.100 \\
\hline$b 5$ & -2.000 & -1.748 & $12.6 \%$ & 0.131 & -1.879 & $6.0 \%$ & 0.170 & 0.047 & 0.176 \\
\hline \multicolumn{10}{|c|}{ Cholesky parameters characterizing the covariance matrix of the $\beta$ vector } \\
\hline$l 11$ & 1.000 & 0.837 & $16.3 \%$ & 0.078 & 0.914 & $8.6 \%$ & 0.105 & 0.026 & 0.109 \\
\hline$l 12$ & -0.500 & -0.398 & $20.4 \%$ & 0.068 & -0.440 & $12.1 \%$ & 0.089 & 0.014 & 0.090 \\
\hline$l 13$ & 0.250 & 0.275 & $9.9 \%$ & 0.090 & 0.269 & $7.5 \%$ & 0.101 & 0.012 & 0.102 \\
\hline$l 14$ & 0.750 & 0.657 & $12.5 \%$ & 0.073 & 0.690 & $8.0 \%$ & 0.094 & 0.017 & 0.095 \\
\hline$l 15$ & 0.000 & 0.011 & $1.1 \%$ & 0.073 & -0.009 & $0.9 \%$ & 0.103 & 0.017 & 0.104 \\
\hline 122 & 0.866 & 0.704 & $18.7 \%$ & 0.080 & 0.745 & $14.0 \%$ & 0.095 & 0.023 & 0.098 \\
\hline 123 & 0.433 & 0.314 & $27.4 \%$ & 0.095 & 0.366 & $15.6 \%$ & 0.106 & 0.014 & 0.107 \\
\hline 124 & -0.144 & -0.075 & $47.8 \%$ & 0.065 & -0.098 & $32.2 \%$ & 0.086 & 0.014 & 0.088 \\
\hline 125 & 0.000 & 0.011 & $1.1 \%$ & 0.080 & 0.023 & $2.3 \%$ & 0.103 & 0.018 & 0.104 \\
\hline 133 & 0.866 & 0.764 & $11.8 \%$ & 0.106 & 0.775 & $10.5 \%$ & 0.120 & 0.028 & 0.124 \\
\hline 134 & 0.237 & 0.163 & $31.1 \%$ & 0.076 & 0.164 & $30.7 \%$ & 0.097 & 0.017 & 0.098 \\
\hline 135 & 0.000 & -0.015 & $1.5 \%$ & 0.093 & -0.047 & $4.7 \%$ & 0.133 & 0.021 & 0.135 \\
\hline 144 & 0.601 & 0.286 & $52.4 \%$ & 0.095 & 0.498 & $17.1 \%$ & 0.110 & 0.027 & 0.113 \\
\hline 145 & 0.000 & 0.184 & $18.4 \%$ & 0.104 & 0.026 & $2.6 \%$ & 0.176 & 0.043 & 0.182 \\
\hline 155 & 1.000 & 0.824 & $17.6 \%$ & 0.091 & 0.871 & $12.9 \%$ & 0.139 & 0.042 & 0.145 \\
\hline \multicolumn{2}{|c|}{$\begin{array}{l}\text { Overall Mean Value } \\
\text { Across Parameters } \\
\end{array}$} & - & $17.8 \%$ & 0.090 & - & $10.6 \%$ & 0.116 & 0.025 & 0.119 \\
\hline \multicolumn{2}{|c|}{ Mean Time } & \multicolumn{3}{|c|}{192.65} & \multicolumn{5}{|c|}{24.41} \\
\hline \multicolumn{2}{|c|}{ Std. dev of Time } & \multicolumn{3}{|c|}{52.31} & \multicolumn{5}{|c|}{7.81} \\
\hline \multicolumn{2}{|c|}{ \% of Runs Converged } & \multicolumn{3}{|c|}{$50 \%$} & \multicolumn{5}{|c|}{$100 \%$} \\
\hline
\end{tabular}


Table 3a: Evaluation of the ability to recover true parameters for the panel intra-individual and inter-individual random coefficients diagonal case

\begin{tabular}{|c|c|c|c|c|c|c|}
\hline \multirow{3}{*}{ Parameter } & \multirow{3}{*}{$\begin{array}{l}\text { True } \\
\text { Value }\end{array}$} & \multicolumn{5}{|c|}{ MACML Method } \\
\hline & & \multicolumn{2}{|c|}{ Parameter Estimates } & \multicolumn{3}{|c|}{ Standard Error Estimates } \\
\hline & & $\begin{array}{c}\text { Mean } \\
\text { Estimate }\end{array}$ & $\begin{array}{l}\text { Absolute } \\
\text { Percentage } \\
\text { Bias }\end{array}$ & $\begin{array}{l}\text { Asymptotic } \\
\text { Standard } \\
\text { Error }\end{array}$ & $\begin{array}{l}\text { Approximation } \\
\text { Standard Error }\end{array}$ & $\begin{array}{c}\text { Approximation } \\
\text { Adjusted Asymptotic } \\
\text { Standard Error }\end{array}$ \\
\hline \multicolumn{7}{|c|}{ Mean values of the $\beta$ vector } \\
\hline$b 1$ & 1.500 & 1.341 & $10.6 \%$ & 0.272 & 0.045 & 0.276 \\
\hline$b 2$ & -1.000 & -0.851 & $14.9 \%$ & 0.176 & 0.027 & 0.178 \\
\hline$b 3$ & 2.000 & 1.753 & $12.3 \%$ & 0.350 & 0.057 & 0.355 \\
\hline$b 4$ & 1.000 & 0.913 & $8.7 \%$ & 0.191 & 0.031 & 0.193 \\
\hline$b 5$ & -2.000 & -1.780 & $11.0 \%$ & 0.357 & 0.059 & 0.362 \\
\hline \multicolumn{7}{|c|}{ Standard deviations of the $\beta$ vector } \\
\hline$\sigma 1$ & 1.000 & 0.842 & $15.8 \%$ & 0.191 & 0.038 & 0.195 \\
\hline$\sigma 2$ & 1.000 & 0.815 & $18.5 \%$ & 0.179 & 0.028 & 0.181 \\
\hline$\sigma 3$ & 1.000 & 0.865 & $13.5 \%$ & 0.195 & 0.035 & 0.198 \\
\hline$\sigma 4$ & 1.000 & 0.864 & $13.6 \%$ & 0.188 & 0.032 & 0.190 \\
\hline$\sigma 5$ & 1.000 & 0.877 & $12.3 \%$ & 0.197 & 0.038 & 0.200 \\
\hline$\tilde{\sigma} 1$ & 1.000 & 0.919 & $8.1 \%$ & 0.243 & 0.037 & 0.245 \\
\hline$\tilde{\sigma}_{2}$ & 1.000 & 0.917 & $8.3 \%$ & 0.239 & 0.044 & 0.243 \\
\hline$\tilde{\sigma} 3$ & 1.000 & 0.819 & $18.1 \%$ & 0.235 & 0.072 & 0.246 \\
\hline$\tilde{\sigma} 4$ & 1.000 & 0.856 & $14.4 \%$ & 0.230 & 0.036 & 0.233 \\
\hline$\tilde{\sigma} 5$ & 1.000 & 0.909 & $9.1 \%$ & 0.248 & 0.038 & 0.251 \\
\hline \multicolumn{2}{|c|}{$\begin{array}{l}\text { Overall Mean Value } \\
\text { Across Parameters }\end{array}$} & & $12.6 \%$ & 0.233 & 0.041 & 0.236 \\
\hline \multicolumn{2}{|l|}{ Mean Time } & \multicolumn{5}{|c|}{22.82} \\
\hline \multicolumn{2}{|c|}{ Std. dev of Time } & \multicolumn{5}{|c|}{4.53} \\
\hline \multicolumn{2}{|c|}{$\%$ of Runs Converged } & \multicolumn{5}{|c|}{$100 \%$} \\
\hline
\end{tabular}


Table 3b: Evaluation of the ability to recover true parameters for the panel intra-individual and inter-individual random coefficients non-diagonal case

\begin{tabular}{|c|c|c|c|c|c|c|}
\hline \multirow{3}{*}{ Parameter } & \multirow{3}{*}{$\begin{array}{l}\text { True } \\
\text { Value }\end{array}$} & \multicolumn{5}{|c|}{ MACML Method } \\
\hline & & \multicolumn{2}{|c|}{ Parameter Estimates } & \multicolumn{3}{|c|}{ Standard Error Estimates } \\
\hline & & $\begin{array}{c}\text { Mean } \\
\text { Estimate }\end{array}$ & $\begin{array}{c}\text { Absolute } \\
\text { Percentage } \\
\text { Bias }\end{array}$ & $\begin{array}{l}\text { Asymptotic } \\
\text { Standard } \\
\text { Error }\end{array}$ & $\begin{array}{l}\text { Approximation } \\
\text { Standard Error }\end{array}$ & $\begin{array}{c}\text { Approximation } \\
\text { Adjusted Asymptotic } \\
\text { Standard Error }\end{array}$ \\
\hline \multicolumn{7}{|c|}{ Mean values of the $\beta$ vector } \\
\hline$b 1$ & 1.500 & 1.430 & $4.6 \%$ & 0.288 & 0.028 & 0.289 \\
\hline$b 2$ & -1.000 & -0.936 & $6.4 \%$ & 0.200 & 0.019 & 0.201 \\
\hline$b 3$ & 2.000 & 1.925 & $3.7 \%$ & 0.383 & 0.037 & 0.385 \\
\hline$b 4$ & 1.000 & 0.932 & $6.8 \%$ & 0.195 & 0.018 & 0.196 \\
\hline$b 5$ & -2.000 & -1.903 & $4.8 \%$ & 0.386 & 0.036 & 0.388 \\
\hline \multicolumn{7}{|c|}{ Cholesky parameters characterizing the covariance matrix of the $\beta$ vector } \\
\hline$l 11$ & 1.000 & 0.940 & $6.0 \%$ & 0.214 & 0.023 & 0.215 \\
\hline 112 & -0.500 & -0.548 & $9.7 \%$ & 0.164 & 0.022 & 0.165 \\
\hline$l 13$ & 0.250 & 0.188 & $24.6 \%$ & 0.143 & 0.026 & 0.145 \\
\hline$l 14$ & 0.750 & 0.771 & $2.8 \%$ & 0.204 & 0.026 & 0.205 \\
\hline$l 15$ & 0.000 & 0.103 & $10.3 \%$ & 0.142 & 0.026 & 0.145 \\
\hline 122 & 0.866 & 0.694 & $19.9 \%$ & 0.181 & 0.026 & 0.183 \\
\hline 123 & 0.433 & 0.332 & $23.4 \%$ & 0.195 & 0.039 & 0.199 \\
\hline 124 & -0.144 & -0.030 & $79.2 \%$ & 0.170 & 0.031 & 0.172 \\
\hline 125 & 0.000 & 0.078 & $7.8 \%$ & 0.183 & 0.037 & 0.187 \\
\hline 133 & 0.866 & 0.810 & $6.5 \%$ & 0.209 & 0.034 & 0.212 \\
\hline 134 & 0.237 & 0.167 & $29.5 \%$ & 0.178 & 0.033 & 0.182 \\
\hline 135 & 0.000 & 0.003 & $0.3 \%$ & 0.193 & 0.042 & 0.197 \\
\hline 144 & 0.601 & 0.392 & $34.8 \%$ & 0.223 & 0.035 & 0.226 \\
\hline 145 & 0.000 & -0.168 & $16.8 \%$ & 0.555 & 0.074 & 0.560 \\
\hline 155 & 1.000 & 0.773 & $22.7 \%$ & 0.408 & 0.060 & 0.413 \\
\hline
\end{tabular}


Table 3b: (Continued) Evaluation of the ability to recover true parameters for the panel intra-individual and inter-individual random coefficients non-diagonal case

\begin{tabular}{|c|c|c|c|c|c|c|}
\hline \multirow{3}{*}{ Parameter } & \multirow{3}{*}{$\begin{array}{l}\text { True } \\
\text { Value }\end{array}$} & \multicolumn{5}{|c|}{ MACML Method } \\
\hline & & \multicolumn{2}{|c|}{ Parameter Estimates } & \multicolumn{3}{|c|}{ Standard Error Estimates } \\
\hline & & $\begin{array}{c}\text { Mean } \\
\text { Estimate }\end{array}$ & $\begin{array}{c}\text { Absolute } \\
\text { Percentage } \\
\text { Bias }\end{array}$ & $\begin{array}{l}\text { Asymptotic } \\
\text { Standard } \\
\text { Error }\end{array}$ & $\begin{array}{l}\text { Approximation } \\
\text { Standard Error }\end{array}$ & $\begin{array}{c}\text { Approximation } \\
\text { Adjusted Asymptotic } \\
\text { Standard Error }\end{array}$ \\
\hline \multicolumn{7}{|c|}{ Cholesky parameters characterizing the covariance matrix of the $\tilde{\beta}$ vector } \\
\hline$\tilde{l} 11$ & 1.000 & 1.033 & $3.3 \%$ & 0.254 & 0.027 & 0.255 \\
\hline$\tilde{l} 12$ & 0.000 & -0.007 & $0.7 \%$ & 0.170 & 0.023 & 0.172 \\
\hline$\tilde{l} 13$ & 0.000 & 0.043 & $4.3 \%$ & 0.188 & 0.030 & 0.190 \\
\hline$\tilde{l} 14$ & 0.000 & 0.030 & $3.0 \%$ & 0.177 & 0.023 & 0.178 \\
\hline$\tilde{l} 15$ & 0.000 & -0.008 & $0.8 \%$ & 0.183 & 0.027 & 0.185 \\
\hline$\tilde{l} 22$ & 1.000 & 0.973 & $2.7 \%$ & 0.249 & 0.028 & 0.251 \\
\hline$\tilde{l} 23$ & 0.500 & 0.527 & $5.3 \%$ & 0.213 & 0.031 & 0.215 \\
\hline$\tilde{l} 24$ & 0.500 & 0.415 & $17.0 \%$ & 0.197 & 0.026 & 0.199 \\
\hline$\tilde{l} 25$ & 0.500 & 0.422 & $15.7 \%$ & 0.198 & 0.029 & 0.200 \\
\hline$\tilde{l} 33$ & 0.866 & 0.798 & $7.9 \%$ & 0.272 & 0.036 & 0.275 \\
\hline$\tilde{l} 34$ & 0.289 & 0.361 & $25.0 \%$ & 0.265 & 0.039 & 0.268 \\
\hline$\tilde{l} 35$ & 0.289 & 0.390 & $35.2 \%$ & 0.271 & 0.045 & 0.274 \\
\hline$\tilde{l} 44$ & 0.817 & 0.720 & $11.8 \%$ & 0.305 & 0.033 & 0.306 \\
\hline$\tilde{l} 45$ & 0.204 & 0.285 & $39.4 \%$ & 0.300 & 0.045 & 0.303 \\
\hline$\tilde{l} 55$ & 0.791 & 0.460 & $41.8 \%$ & 0.307 & 0.077 & 0.316 \\
\hline \multicolumn{2}{|c|}{$\begin{array}{l}\text { Overall Mean Value } \\
\text { Across Parameters }\end{array}$} & & $15.3 \%$ & 0.239 & 0.034 & 0.241 \\
\hline \multicolumn{2}{|l|}{ Mean Time } & \multicolumn{5}{|c|}{46.50} \\
\hline \multicolumn{2}{|c|}{ Std. dev of Time } & \multicolumn{5}{|c|}{8.83} \\
\hline \multicolumn{2}{|c|}{$\%$ of Runs Converged } & \multicolumn{5}{|c|}{$100 \%$} \\
\hline
\end{tabular}

